\title{
Fixed-dose combination products and unintended drug interactions: urgent need for pharmacogenetic evaluation
}

\author{
"We recommend that sponsors conduct clinical pharmacogenetic \\ studies during the initial development phase of fixed-dose \\ combinations and voluntarily submit findings to the regulatory \\ agency in order to validate scientific arguments in favor of safety and \\ effectiveness."
}

Keywords: adverse events $\bullet$ drug interactions $\bullet$ fixed-dose combinations $\bullet$ pharmacogenetics - regulatory policy

Over the past $10+$ years, we have been witness of a rapid progression in our understanding of the role of inheritance on the observed individual variance in drug responses and metabolism [1,2]. A relatively higher abundance of genetic polymorphisms of clinical interest are described at the level of drug metabolisms where up to $40 \%$ of phase I biotransformation of medically used drugs, including both drug elimination and activation of prodrugs, is mediated by enzymes with polymorphisms known to have a significant impact on their activity in vivo.

Well-known variable isoenzymes are the cytochrome-P450 enzymes CYP2D6, CYP2C9 and CYP2C19, which all constitute valid biomarkers for predicting clinical outcomes in humans [3-9]. Among the drugs with current pharmacogenetic-driven relabeling, the metabolizing enzymes are in a majority accounting for $80 \%$ of such revised labels by the US FDA (e.g., Warfarin and Irinotecan, among others). New genomic technologies are incorporated into the clinical development programs sponsored by big pharmaceutical companies. Guidance for industry was first issued in 2006 (reissued in 2007) encouraging sponsors to submit pharmacogenomic data in support of an application approval by the FDA [10]. In addition, a guideline on the use of pharmacogenetics in the evaluation of medicinal products (EMA/CHMP/37646/2009) was published in December 2011 by the Committee for Medicinal Products for Human Use of the EMA [11].

\section{Statement of the problem}

These advances have not been applied to fixeddose combinations (FDCs) or co-packaged versions of previously approved drug products [12-14]. The number of FDCs currently developed by pharmaceutical companies is considerable, and their future is looming large, as convenience and savings drive the bundling of different product classes into combination pills. Although some reports in the scientific literature suggest a better compliance as well as greater benefits with FDCs [15-19], it might also be a direct consequence of patent expirations for the individual components in the market. Since FDCs can indeed be patented as a novel chemical entity, they are developed as a way to extend proprietary rights and marketability of their own drug products, even after individual active ingredients and some medical uses thereof are off-patent.

For the purpose of this commentary, the term FDC drug product is defined as: "a drug product that according to its proposed labeling is for use only with another specified drug product where both are required to achieve the intended use, indication, or effect at a fixed ratio of doses. Usually, they are administered as a finished pharmaceutical product that contains two or more therapeutically

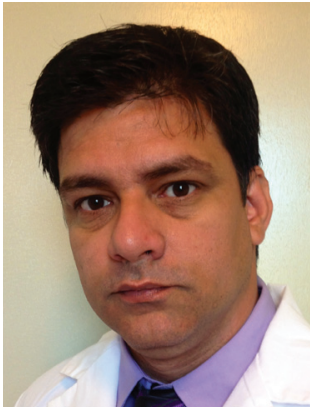

Jorge Duconge Author for correspondence: School of Pharmacy, University of Puerto Rico, Medical Sciences Campus, PO Box 365067, San Juan, PR 00936, USA

Tel.: +1 7877582525 (ext. 5312)

Fax: +1 7877672796

jorge.duconge@upr.edu

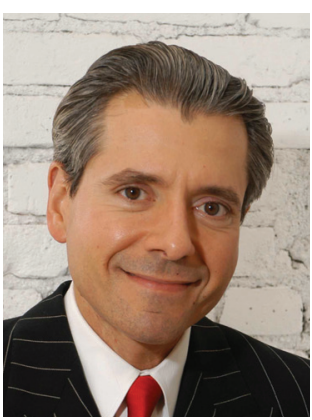

Gualberto Ruaño Genomas Inc., Hartford, CT 06106, USA

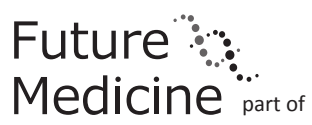
fsg 
active entities. This term does not include co-packaged versions of previously approved drug products." Although FDCs were initially developed to target a single illness (i.e., antiretroviral combinations to fight AIDS), today they can also be used against multiple diseases or medical conditions [20-23]. For example, Caduet $^{\circledR}$ (amlodipine besylate $2.5 \mathrm{mg}$ /atorvastatin calcium $10 \mathrm{mg}$ ) is prescribed to treat hypercholesterolemia and high blood pressure and thus lower the risk of heart attacks and other deleterious cardiovascular events. Because FDCs would involve active ingredients that were regulated under different regulatory policies, they raise some challenging regulatory concerns. Differences in regulatory pathways can influence the regulatory processes for all aspects of product development, including clinical investigation.

\section{"At present, an increasing proportion of drugs selected for development as fixed-dose combinations are metabolized (or requires activation) by polymorphic enzymes in the body."}

\section{Safety of FDCs \& role of pharmacogenetics}

One misconception in the development of FDCs is the erroneous perception that their active ingredients are not likely to give rise to any significant 'undue' side event (including therapeutic failures, adverse effects or drug interactions with each other). It is because their applications are fully revised by the regulatory bodies and are usually formulated in combination products at doses of the active ingredients that are less than if they were prescribed separately. Lack of enough supportive data from proper pharmacogenetic studies of FDCs and their separate ingredients could nullify such an expectation.

At present, an increasing proportion of drugs selected for development as FDCs are metabolized (or requires activation) by polymorphic enzymes in the body. However, there is very little or no knowledge on any clinically relevant impact of their pharmacogenetics once the product is administered to the patient as the combination of two or more active ingredients and the potential for genotype-dependent drug interactions (Supplementary Table 1). This is particularly important for products under development when the altered enzyme(s) is (are) either an essential part of the primary pathway for eliminating both drugs from the body or a critical step in the onset of their drug actions. Either way, the expected therapeutic outcomes will be compromised due to an increased risk for drugs accumulation and toxicity or for subtherapeutic levels and inactivity, respectively. Not to mention when both drugs compete for the same metabolic pathway. Another aspect of this potential interaction could be revealed if the exposure to one of the drugs in the combination gives rise to either an induction or an inhibition effect on the polymorphic enzyme. In this context, an intermediate metabolizer could turn out to be a poor metabolizer instead (phenocopy). Accordingly, pharmacogenetic-driven drug interactions at this pharmacokinetic level (metabolism) need further attention given their potential clinical consequences. That is, it is necessary to assess the effect of the different allelic variants on prodrug activations or drug clearance through the specific metabolic pathway related to the polymorphic gene(s).

FDC that does not meet a patient's dose requirement (e.g., a poor metabolizer), with the patient receiving too much (or too little) of one of the active ingredients in the combination, will further limit the clinician's ability to individualize the patient's dosing regimen.

\section{Nuedexta ${ }^{\circledR}$ : a case for deliberate drug interactions?}

Approved by the US FDA in 2010, Nuedexta ${ }^{\circledR}$ (Avanir Pharmaceuticals, CA, USA) is used to treat pseudobulbar affect (PBA), involuntary outbursts of crying or laughing in patients with certain neurological disorders, including multiple sclerosis and amyotrophic lateral sclerosis (ALS, or Lou Gehrig's disease). Nuedexta may also be used for off-label purposes in psychiatry. Developed by Avanir Pharmaceuticals, Nuedexta is a combination product containing $20 \mathrm{mg}$ dextromethorphan hydrobromide (an uncompetitive NMDA receptor antagonist and sigma-1 agonist) and $10 \mathrm{mg}$ quinidine sulfate (a CYP450 2D6 inhibitor) per capsule. Dextromethorphan affects the signals in the brain that trigger cough reflex and is generally used as an antitussive. Pharmacokinetically, dextromethorphan is a classic, major CYP2D6 substrate. Quinidine affects the electrical conduction in the heart and is generally used to treat cardiac arrhythmias. Interestingly, while the components of Nuedexta are classical drugs known for decades, their combination and its therapeutic indication are novel.

The quinidine in Nuedexta inhibits CYP2D6 in patients in whom CYP2D6 is not otherwise genetically absent or its activity otherwise pharmacologically inhibited. Because of this effect on CYP2D6, accumulation of parent drug and/or failure of active metabolite formation may increase the risk of side effects and/or reduce the efficacy of drugs used concomitantly with Nuedexta that are metabolized by CYP2D6. The quinidine component of Nuedexta is deliberately intended to inhibit CYP2D6, so that higher exposure to dextromethorphan can be achieved compared with when dextromethorphan is given alone. In fact, the quinidine component of Nuedexta is not therapeutic at all, but expected to contribute to the effectiveness of Nuedexta.

The drug label contains an explicit pharmacogenomics section stating: "The quinidine component of 
Nuedexta is intended to inhibit CYP2D6 so that higher exposure to dextromethorphan can be achieved compared with when dextromethorphan is given alone. In those patients who may be at risk of significant toxicity due to quinidine, genotyping to determine if they are PMs should be considered prior to making the decision to treat with Nuedexta." [24].

This guidance for pharmacogenetic testing in the drug label is noteworthy as a safeguard on the broad use of the drug for all patients. In CYP2D6 null or poor metabolizers, quinidine will have little if any CYP2D6 enzyme to inhibit, which would result in liberation of this agent in the circulation, to induce its pharmacological effect as arrhythmic agent, potentially dangerous. The same problem is likely in patients taking other psychiatric drugs that are dual substrates and inhibitors of CYP2D6, such as amitriptyline, fluoxetine and paroxetine. When Nuedexta is prescribed with drugs that inhibit or are extensively metabolized by CYP2D6, consideration should be given to initiating treatment with a lower dose. In ultrarapid metabolizers, quinidine is likely to have little effect at the dosage included in Nuedexta, thus disrupting the pharmacokinetic rationale for the drug combination in the first place.

For Nuedexta, both null/poor and ultrarapid metabolizers, patients at either extreme of the CYP2D6 functional spectrum, are being deliberately exposed to a dangerous drug combination and interaction. It would appear that pharmacogenetic testing should be a requirement for prescribing this drug, not merely a suggestion. The deliberate drug interaction pursued for Nuedexta works only for patients with normal (average) CYP2D6 functional status.

\section{Conclusion}

The present work discusses the urgent need for pharmacogenetic (PGt) evaluations of FDCs or co-pack- aged versions of previously approved drug products, as their growing appeal will raise some challenging regulatory concerns. An example of the clinical consequences of inappropriate evaluations of these PGt risks is discussed. We concluded that the lack of enough supportive data from proper PGt studies of FDCs could nullify any expectation of developing safe products. We recommend that sponsors conduct clinical PGt studies during the initial development phase of FDCs and voluntarily submit findings to the regulatory agency in order to validate scientific arguments in favor of safety and effectiveness.

\section{Future perspective}

A rational approach is to be adopted by key players (i.e., industry, regulatory agency and researchers) through a thorough evaluation of the clinical relevance of any PGt effect on drug exposure as well as the benefits of applying individual genotyping in future clinical use of FDC products. This information is also expected to be used for decision-making on projected dosing schemes as well as to be reflected in drug labeling.

\section{Supplementary data}

To view the supplementary data that accompany this paper, please visit the journal website at: www.futuremedicine.com/ doi/full/10.2217/PGS.15.123

\section{Financial \& competing interests disclosure}

This article was funded in part by the grant \#SC1 HL123911 from the National Heart, Lung and Blood Institute (NHLBI, $\mathrm{NIH}$ ). G Ruaño is founder and President of Genomas Inc. The authors have no other relevant affiliations or financial involvement with any organization or entity with a financial interest in or financial conflict with the subject matter or materials discussed in the manuscript apart from those disclosed.

No writing assistance was utilized in the production of this manuscript.

\section{Executive summary}

- Currently, there is a lack of enough supportive data from proper pharmacogenetic (PGt) studies of fixed-dose combinations (FDCs) or co-packaged versions of previously approved drug products.

- Clinical PGt studies of FDCs should be conducted by sponsors during their initial development phases.

- Voluntary submission of collected PGt data to the regulatory agency should be encouraged in order to support scientific arguments in favor of safety and effectiveness of FDCs.

- The Nuedexta ${ }^{\circledR}$ example, portrayed as a case for deliberate PGt-driven drug interactions, makes a strong case for incorporating PGt evaluations in future regulatory policies.

\section{References}

Papers of special note have been highlighted as:

- of interest; $\bullet$ of considerable interest

1 Wei CY, Lee MT, Chen YT. Pharmacogenomics of adverse drug reactions: implementing personalized medicine. Hum. Mol. Genet. 21(R1), R58-R65 (2012).
2 Severino G, Del Zompo M. Adverse drug reactions: role of pharmacogenomics. Pharmacol. Res. 49, 363-373 (2004).

-• Excellent review with several examples of genetic polymorphisms affecting drug kinetics, drug toxicity and hypersensitivity related to adverse drug reactions. 
3 Johnson JA, Gong L, Whirl-Carrillo M et al. Clinical Pharmacogenetics Implementation Consortium guidelines for CYP2C9 and VKORC1 genotypes and warfarin dosing. Clin. Pharmacol. Ther. 90, 625-629 (2011).

4 Scott SA, Sangkuhl K, Gardner EE et al. Clinical Pharmacogenetics Implementation Consortium guidelines for cytochrome P450-2C19 (CYP2C19) genotype and clopidogrel therapy. Clin. Pharmacol. Ther. 90, 328-332 (2011).

5 Crews KR, Gaedigk A, Dunnenberger HM et al. Clinical Pharmacogenetics Implementation Consortium guidelines for cytochrome P450 2D6 genotype and codeine therapy: 2014 update. Clin. Pharmacol. Ther. 95, 376-382 (2014).

6 Goldstein J. Clinical relevance of genetic polymorphisms in the human CYP2C subfamily. Br. J. Clin. Pharmacol. 52(4), 349-355 (2001).

- Wide-ranging and very useful review on the clinical relevance of genetic polymorphisms in the human CYP2C subfamily.

7 Van Booven D, Marsh S, McLeod H et al. Cytochrome P450 2C9-CYP2C9. Pharmacogenet. Genomics 20(4), 277-81 (2010).

8 Scott S, Sangkuhl K, Shuldiner AR et al. PharmGKB summary: very important pharmacogene information for cytochrome P450, family 2, subfamily C, polypeptide 19. Pharmacogenet. Genomics 22(2), 159-165 (2012).

9 Owen RP, Sangkuhl K, Klein TE, Altman RB. Cytochrome P450 2D6. Pharmacogenet. Genomics 19(7), 559-562 (2009).

10 US FDA. Guidance for industry: pharmacogenomic data submissions - companion guidance. US Department of Health and Human Services, Food and Drug Administration (2007).

www.fda.gov/downloads/Drugs/GuidanceCompliance

11 EMA. Guideline on the use of pharmacogenetic methodologies in the pharmacokinetic evaluation of medicinal products. EMA/CHMP/37646/2009. Committee for Medicinal Products for Human Use, European Medicine Agency (2012).

www.ema.europa.eu/docs/en_GB/document_library

12 Sica DA. Rationale for fixed-dose combinations in the treatment of hypertension: the cycle repeats. Drugs 62(3), 443-462 (2002).

- Establishes rationale for fixed-dose combinations in hypertension therapy.

13 Leichter SB, Thomas S. Combination medications in diabetes care: an opportunity that merits more attention. Clin. Diabetes 21(4), 175-178 (2003).
- Establishes rationale for fixed-dose combinations in diabetes therapy.

14 Podolsky SH, Greene JA. Combination drugs - hype, harm, and hope. N. Engl. J. Med. 356(6), 488-491 (2011).

-. Excellent perspective on the historical context in which the role of government and industry in reforming regulatory environment for the development of fixed drug combinations should be discussed.

15 Hutchins V, Zhang B, Fleurence RL, Krishnarajah G, Graham J. A systematic review of compliance, treatment satisfaction and costs, in fixed-dose combination regimens in Type 2 diabetes. Curr. Med. Res. Opin. 27(6), 1157-1168 (2011).

16 Gupta AK, Arshad S, Poulter NR. Compliance, safety, and effectiveness of fixed-dose combinations of antihypertensive agents: a meta-analysis. Hypertension 55, 399-407 (2010).

17 Deharo P, Quilici J, Bonnet G et al. Fixed-dose aspirinclopidogrel combination enhances compliance to aspirin after acute coronary syndrome. Int. J. Cardiol. 172(1), e1-e2 (2014).

18 Thom S, Poulter N, Field J et al. UMPIRE Collaborative Group. Effects of a fixed-dose combination strategy on adherence and risk factors in patients with or at high risk of CVD: the UMPIRE randomized clinical trial. JAMA 310(9), 918-929 (2013).

19 Bangalore S, Kamalakkannan G, Parkar S, Messerli FH. Fixed-dose combinations improve medication compliance: a meta-analysis. Am. J. Med. 120, 713-719 (2007).

- Good review on data comparison of fixed-dose combination with free-drug regimen to improve patient's medication compliance.

20 Woodcock J, Griffin JP, Behrman RE. Development of novel combination therapies. N. Engl. J. Med. 364(11), 985-987 (2011).

21 PILL Collaborative Group, Rodgers A, Patel A et al. An international randomized placebo-controlled trial of a fourcomponent combination pill ("polypill") in people with raised cardiovascular risk. PLoS ONE 6(5), e19857 (2011).

22 US FDA. Guidance for industry - co-development of two or more unmarketed investigational drugs for use in combination

www.fda.gov/downloads/Drugs/GuidanceCompliance

23 Drugs at FDA resources page. www.accessdata.fda.gov/scripts/cder/drugsatfda/index.cfm

24 Nuedexta 20/10 Package Label. Avanir Pharmaceuticals, Inc. 5.8 CYP2D6 poor metabolizers (2010). www.drugs.com/pro/nuedexta.html 